\title{
AN OPERATOR-THEORETICAL PROOF FOR THE SECOND-ORDER PHASE TRANSITION IN THE BCS-BOGOLIUBOV MODEL OF SUPERCONDUCTIVITY
}

\author{
Shuji WATANABE
}

(Received 18 December 2018)

\begin{abstract}
We show that the transition from a normal conducting state to a superconducting state is a second-order phase transition in the BCS-Bogoliubov model of superconductivity from the viewpoint of operator theory. Here we have no magnetic field. Moreover we obtain the exact and explicit expression for the gap in the specific heat at constant volume at the transition temperature. To this end, we have to differentiate the thermodynamic potential with respect to the temperature twice. Since there is a solution to the BCS-Bogoliubov gap equation in the form of the thermodynamic potential, we have to differentiate the solution with respect to the temperature twice. Therefore, we need to show that the solution to the BCS-Bogoliubov gap equation is differentiable with respect to the temperature twice, as well as its existence and uniqueness. We carry out its proof on the basis of fixed point theorems.
\end{abstract}

\section{Introduction and preliminaries}

In this paper we show that the transition from a normal conducting state to a superconducting state is a second-order phase transition in the BCS-Bogoliubov model of superconductivity from the viewpoint of operator theory. Here we have no magnetic field. Moreover we obtain the exact and explicit expression for the gap in the specific heat at constant volume at the transition temperature. To this end, we have to differentiate the thermodynamic potential (see (1.6)) with respect to the absolute temperature $T$ twice. Since there is a solution to the BCS-Bogoliubov gap equation in the form of the thermodynamic potential, we have to differentiate the solution with respect to the temperature $T$ twice. Therefore, we need to show that the solution to the BCS-Bogoliubov gap equation is differentiable with respect to the temperature $T$ twice, as well as its existence and uniqueness. We carry out its proof on the basis of fixed point theorems.

The BCS-Bogoliubov gap equation $[2,4]$ is a nonlinear integral equation:

$$
u(T, x)=\int_{\varepsilon}^{\hbar \omega_{D}} \frac{U(x, \xi) u(T, \xi)}{\sqrt{\xi^{2}+u(T, \xi)^{2}}} \tanh \frac{\sqrt{\xi^{2}+u(T, \xi)^{2}}}{2 T} d \xi, \quad T \geq 0, \quad \varepsilon \leq x \leq \hbar \omega_{D},
$$

2010 Mathematics Subject Classification: Primary 45G10, 47H10, 47N50, 82D55.

Keywords: BCS-Bogoliubov gap equation; nonlinear integral equation; second-order phase transition; superconductivity. 
where the solution $u$ is a function of the absolute temperature $T$ and the energy $x$. The constant $\omega_{D}>0$ stands for the Debye angular frequency. The potential $U$ satisfies $U(x, \xi)>$ 0 at all $(x, \xi) \in\left[\varepsilon, \hbar \omega_{D}\right]^{2}$.

In (1.1) we need to introduce a cutoff $\varepsilon>0$, which is sufficiently small and fixed. In the original BCS-Bogoliubov gap equation, one sets $\varepsilon=0$. However we introduce a very small $\varepsilon>0$. See Remark 1.12 for the reason why we need to introduce $\varepsilon>0$.

In (1.1) we consider the solution $u$ as a function of the absolute temperature $T$ and the energy $x$. Accordingly, we deal with the integral with respect to the energy $\xi$ in (1.1). Sometimes one considers the solution $u$ as a function of the absolute temperature and the wave vector. Accordingly, instead of the integral in (1.1), one deals with the integral with respect to the wave vector over the three-dimensional Euclidean space $\mathbb{R}^{3}$. Odeh [12], and Billard and Fano [3] established the existence and uniqueness of the solution to the BCSBogoliubov gap equation for $T=0$, and Vansevenant [13] for $T \geq 0$. Bach, Lieb and Solovej [1] studied the gap equation in the Hubbard model for a constant potential, and showed that its solution is strictly decreasing with respect to the temperature. Frank, Hainzl, Naboko and Seiringer [5] studied the asymptotic behavior of the transition temperature (the critical temperature) at weak coupling. Hainzl, Hamza, Seiringer and Solovej [6] proved that the existence of a positive solution to the BCS-Bogoliubov gap equation is equivalent to the existence of a negative eigenvalue of a certain linear operator, and showed the existence of a transition temperature. Hainzl and Seiringer [7] obtained upper and lower bounds on the transition temperature and the energy gap for the BCS-Bogoliubov gap equation. For interdisciplinary reviews of the BCS-Bogoliubov model of superconductivity, see Kuzemsky [8, 9]; see also Kuzemsky [10, Chs 26 and 29].

We define a nonlinear integral operator $A$ by

$$
A u(T, x)=\int_{\varepsilon}^{\hbar \omega_{D}} \frac{U(x, \xi) u(T, \xi)}{\sqrt{\xi^{2}+u(T, \xi)^{2}}} \tanh \frac{\sqrt{\xi^{2}+u(T, \xi)^{2}}}{2 T} d \xi .
$$

Here the right-hand side of this equality is exactly the right-hand side of the BCS-Bogoliubov gap equation (1.1). Since the solution to the BCS-Bogoliubov gap equation is a fixed point of our operator $A$, we apply fixed point theorems to our operator $A$.

Let $U_{1}>0$ be a positive constant and set $U(x, \xi)=U_{1}$ at all $(x, \xi) \in\left[\varepsilon, \hbar \omega_{D}\right]^{2}$. Then the solution to the BCS-Bogoliubov gap equation becomes a function of the temperature $T$ only, and we denote the solution by $\Delta_{1}$. Accordingly, the BCS-Bogoliubov gap equation (1.1) is reduced to the simple gap equation [2]

$$
1=U_{1} \int_{\varepsilon}^{\hbar \omega_{D}} \frac{1}{\sqrt{\xi^{2}+\Delta_{1}(T)^{2}}} \tanh \frac{\sqrt{\xi^{2}+\Delta_{1}(T)^{2}}}{2 T} d \xi, \quad 0 \leq T \leq \tau_{1},
$$

where the temperature $\tau_{1}>0$ is defined by (see [2])

$$
1=U_{1} \int_{\varepsilon}^{\hbar \omega_{D}} \frac{1}{\xi} \tanh \frac{\xi}{2 \tau_{1}} d \xi
$$

See also Niwa [11] and Ziman [17].

As is well known, physicists and engineers studying superconductivity always assume that there is a unique non-negative solution $\Delta_{1}$ to the simple gap equation (1.3), that the 
solution $\Delta_{1}$ is continuous and strictly decreasing with respect to the temperature $T$, and that the solution $\Delta_{1}$ is of class $C^{2}$ with respect to the temperature $T$, and so on. But, as far as the present author knows, there is no mathematical proof for these assumptions imposed in the BCS-Bogoliubov model. Applying the implicit function theorem to the simple gap equation (1.3), we obtain the following proposition that indeed gives a mathematical proof for these assumptions.

Proposition 1.1. [14, Proposition 1.2] Let $U_{1}>0$ be a positive constant and set $U(x, \xi)=U_{1}$ at all $(x, \xi) \in\left[\varepsilon, \hbar \omega_{D}\right]^{2}$. Set

$$
\Delta=\frac{\sqrt{\left(\hbar \omega_{D}-\varepsilon e^{1 / U_{1}}\right)\left(\hbar \omega_{D}-\varepsilon e^{\left.-1 / U_{1}\right)}\right.}}{\sinh \left(1 / U_{1}\right)} .
$$

Then there is a unique non-negative solution $\Delta_{1}:\left[0, \tau_{1}\right] \rightarrow[0, \infty)$ to the simple gap equation (1.3) such that the solution $\Delta_{1}$ is continuous and strictly decreasing with respect to the temperature $T$ on the closed interval $\left[0, \tau_{1}\right]$ :

$$
\Delta_{1}(0)=\Delta>\Delta_{1}\left(T_{1}\right)>\Delta_{1}\left(T_{2}\right)>\Delta_{1}\left(\tau_{1}\right)=0, \quad 0<T_{1}<T_{2}<\tau_{1} .
$$

Moreover, the solution $\Delta_{1}$ is of class $C^{2}$ with respect to the temperature $T$ on the interval $\left[0, \tau_{1}\right)$ and satisfies

$$
\Delta_{1}^{\prime}(0)=\Delta_{1}^{\prime \prime}(0)=0 \quad \text { and } \quad \lim _{T \uparrow \tau_{1}} \Delta_{1}^{\prime}(T)=-\infty .
$$

Remark 1.2. We set $\Delta_{1}(T)=0$ at $T>\tau_{1}$. See Figure 1 .

We then introduce another positive constant $U_{2}>0$. Let $0<U_{1}<U_{2}$ and set $U(x, \xi)=$ $U_{2}$ at all $(x, \xi) \in\left[\varepsilon, \hbar \omega_{D}\right]^{2}$. Then a similar discussion implies that for $U_{2}$ there is a unique non-negative solution $\Delta_{2}:\left[0, \tau_{2}\right] \rightarrow[0, \infty)$ to the simple gap equation

$$
1=U_{2} \int_{\varepsilon}^{\hbar \omega_{D}} \frac{1}{\sqrt{\xi^{2}+\Delta_{2}(T)^{2}}} \tanh \frac{\sqrt{\xi^{2}+\Delta_{2}(T)^{2}}}{2 T} d \xi, \quad 0 \leq T \leq \tau_{2} .
$$

Here, $\tau_{2}>0$ is defined by

$$
1=U_{2} \int_{\varepsilon}^{\hbar \omega_{D}} \frac{1}{\xi} \tanh \frac{\xi}{2 \tau_{2}} d \xi
$$

Remark 1.3. We again set $\Delta_{2}(T)=0$ at $T>\tau_{2}$.

LEMMA 1.4. [14, Lemma 1.5]

(a) The inequality $\tau_{1}<\tau_{2}$ holds.

(b) If $0 \leq T<\tau_{2}$, then $\Delta_{1}(T)<\Delta_{2}(T)$. If $T \geq \tau_{2}$, then $\Delta_{1}(T)=\Delta_{2}(T)=0$.

See Figure 1. The function $\Delta_{2}$ has properties similar to those of the function $\Delta_{1}$.

Let us turn to the BCS-Bogoliubov gap equation (1.1). We assume the following condition on $U$ :

$$
U(\cdot, \cdot) \in C\left(\left[\varepsilon, \hbar \omega_{D}\right]^{2}\right), \quad(0<) U_{1} \leq U(x, \xi) \leq U_{2} \quad \text { at all } \quad(x, \xi) \in\left[\varepsilon, \hbar \omega_{D}\right]^{2} .
$$

Let $0 \leq T \leq \tau_{2}$ and fix $T$. We now consider the Banach space $C\left[\varepsilon, \hbar \omega_{D}\right]$ consisting of continuous functions of the energy $x$ only, and deal with the following temperature-dependent subset $V_{T}$ :

$$
V_{T}=\left\{u(T, \cdot) \in C\left[\varepsilon, \hbar \omega_{D}\right]: \Delta_{1}(T) \leq u(T, x) \leq \Delta_{2}(T) \text { at } x \in\left[\varepsilon, \hbar \omega_{D}\right]\right\} .
$$




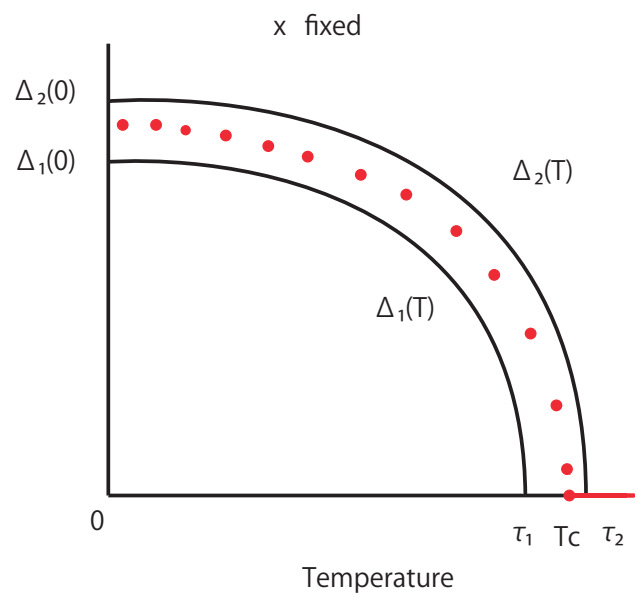

FIGURE 1. The graphs of the functions $\Delta_{1}$ and $\Delta_{2}$ with $x$ fixed. The solution $u_{0}(T, x)$ is between $\Delta_{1}(T)$ and $\Delta_{2}(T)$ for each $T$.

Remark 1.5. The set $V_{T}$ depends on the temperature $T$. See Figure 1.

The following theorem gives another proof of the existence and uniqueness of the nonnegative solution to the BCS-Bogoliubov gap equation, and shows how the solution varies with the temperature.

Theorem 1.6. [14, Theorem 2.2] Assume (1.5) and let $T \in\left[0, \tau_{2}\right]$ be fixed. Then there is a unique non-negative solution $u_{0}(T, \cdot) \in V_{T}$ to the BCS-Bogoliubov gap equation (1.1):

$$
u_{0}(T, x)=\int_{\varepsilon}^{\hbar \omega_{D}} \frac{U(x, \xi) u_{0}(T, \xi)}{\sqrt{\xi^{2}+u_{0}(T, \xi)^{2}}} \tanh \frac{\sqrt{\xi^{2}+u_{0}(T, \xi)^{2}}}{2 T} d \xi, \quad x \in\left[\varepsilon, \hbar \omega_{D}\right] .
$$

Consequently, the solution $u_{0}(T, \cdot)$ with $T$ fixed is continuous with respect to the energy $x$ and varies with the temperature as follows:

$$
\Delta_{1}(T) \leq u_{0}(T, x) \leq \Delta_{2}(T) \quad \text { at }(T, x) \in\left[0, \tau_{2}\right] \times\left[\varepsilon, \hbar \omega_{D}\right] .
$$

See Figure 1 for the graph of the solution $u_{0}$ with the energy $x$ fixed.

Remark 1.7. Let $u_{0}(T, \cdot)$ be as in Theorem 1.6. If there is a point $x_{1} \in\left[\varepsilon, \hbar \omega_{D}\right]$ satisfying $u_{0}\left(T, x_{1}\right)=0$, then $u_{0}(T, x)=0$ at all $x \in\left[\varepsilon, \hbar \omega_{D}\right]$. See [14, Proposition 2.4].

The existence and uniqueness of the transition temperature $T_{c}$ were pointed out in previous papers $[\mathbf{5 - 7 , 1 3}$. In our case, we can define it as follows.

Definition 1.8. Let $u_{0}(T, \cdot)$ be as in Theorem 1.6. Then the transition temperature $T_{c}$ is defined by

$$
T_{c}=\inf \left\{T>0: u_{0}(T, x)=0 \text { at all } x \in\left[\varepsilon, \hbar \omega_{D}\right]\right\} .
$$

Remark 1.9. Let $u_{0}(T, \cdot)$ be as in Theorem 1.6. We then set $u_{0}(T, x)=0$ at all $x \in\left[\varepsilon, \hbar \omega_{D}\right]$ and at $T \geq T_{c}$. The transition temperature $T_{c}$ is the critical temperature that divides normal conductivity and superconductivity, and satisfies $\tau_{1} \leq T_{c} \leq \tau_{2}$. See Figure 1 . 
But Theorem 1.6 tells us nothing about the continuity of the solution $u_{0}$ with respect to the temperature $T$. Applying the Banach fixed point theorem, we then showed in $[\mathbf{1 5}$, Theorem 1.2] that the solution $u_{0}$ is indeed continuous both with respect to the temperature $T$ and with respect to the energy $x$ under the restriction that the temperature $T$ is sufficiently small. See also [16].

In order to discuss the second-order phase transition we need to deal with the thermodynamic potential, as mentioned before. Let us introduce the thermodynamic potential $\Omega$ in the BCS-Bogoliubov model without the magnetic field:

$$
\Omega=-T \ln Z,
$$

where $Z$ denotes the partition function. Throughout this paper we use the unit $k_{B}=1$. Generally speaking, the thermodynamic potential $\Omega$ is a function of the temperature $T$, the chemical potential and the volume of our physical system under consideration. However we fix both the chemical potential and the volume of our physical system, and so we consider the thermodynamic potential $\Omega$ as a function of the temperature $T$ only. We have only to deal with the difference $\Psi$ between the thermodynamic potential corresponding to superconductivity and that corresponding to normal conductivity. The difference $\Psi$ of the thermodynamic potential in the BCS-Bogoliubov model is given by

$$
\begin{aligned}
\Psi(T)= & -2 N_{0} \int_{\varepsilon}^{\hbar \omega_{D}}\left\{\sqrt{\xi^{2}+u_{0}(T, \xi)^{2}}-\xi\right\} d \xi \\
& +N_{0} \int_{\varepsilon}^{\hbar \omega_{D}} \frac{u_{0}(T, \xi)^{2}}{\sqrt{\xi^{2}+u_{0}(T, \xi)^{2}}} \tanh \frac{\sqrt{\xi^{2}+u_{0}(T, \xi)^{2}}}{2 T} d \xi \\
& -4 N_{0} T \int_{\varepsilon}^{\hbar \omega_{D}} \ln \frac{1+e^{-\sqrt{\xi^{2}+u_{0}(T, \xi)^{2}}} / T}{1+e^{-\xi / T}} d \xi, \quad T \in\left[\tau, T_{C}\right],
\end{aligned}
$$

where $N_{0}$ stands for the density of states per unit energy at the Fermi surface, and $u_{0}$ is the solution to the BCS-Bogoliubov gap equation (1.1). Here, $\tau$ is that in Theorem 2.3, and $T_{c}$ is the transition temperature. We define the difference $\Psi$ only on the interval $\left[\tau, T_{c}\right]$ because we are interested in the phase transition at the transition temperature $T_{c}$.

Definition 1.10. The transition from a normal conducting state to a superconducting state at $T=T_{c}$ is a second-order phase transition if the difference $\Psi$ of the thermodynamic potential satisfies the following:
(a) $\Psi \in C^{2}\left[\tau, T_{c}\right]$ and $\Psi\left(T_{c}\right)=0$;
(b) $\frac{\partial \Psi}{\partial T}\left(T_{c}\right)=0$
(c) $\frac{\partial^{2} \Psi}{\partial T^{2}}\left(T_{c}\right) \neq 0$.

Remark 1.11. Condition (a) of Definition 1.10 implies that the thermodynamic potential $\Omega$ is continuous at an arbitrary temperature $T$. Conditions (a) and (b) imply that the entropy $S=-(\partial \Omega / \partial T)$ is also continuous at an arbitrary temperature $T$ and that, as a result, no latent heat is observed at $T=T_{c}$. Hence conditions (a) and (b) imply that the transition at $T=T_{c}$ is not a first-order phase transition. On the other hand, conditions (a) and (c) imply 
that the specific heat at constant volume $C_{V}=-T\left(\partial^{2} \Omega / \partial T^{2}\right)$ is discontinuous at $T=T_{c}$ and that the gap $\Delta C_{V}$ in $C_{V}$ is observed at $T=T_{c}$. Here, the gap $\Delta C_{V}$ at $T=T_{c}$ is given by

$$
\Delta C_{V}=-T_{c} \frac{\partial^{2} \Psi}{\partial T^{2}}\left(T_{c}\right) .
$$

For more details on the entropy and the specific heat at constant volume, see e.g. [2, Section III] or [11, Section 7.7.3].

Remark 1.12. When we differentiate the difference $\Psi$ given by (1.6) with respect to $T$, we have, for example, the term

$$
-N_{0} \int_{\varepsilon}^{\hbar \omega_{D}} \frac{1}{\sqrt{\xi^{2}+u_{0}(T, \xi)^{2}}}\left\{\frac{\partial}{\partial T} u_{0}(T, \xi)^{2}\right\} d \xi .
$$

Note that $u_{0}\left(T_{c}, \xi\right)=0$ at all $\xi$ and that

$$
\left.\frac{\partial}{\partial T} u_{0}(T, \xi)^{2}\right|_{T=T_{c}}=-v(\xi)<0
$$

at all $\xi$. Here the function $v$ is that in Condition (C) of Section 2. The term (1.7) then becomes, at $T=T_{c}$,

$$
N_{0} \int_{\varepsilon}^{\hbar \omega_{D}} \frac{v(\xi)}{\xi} d \xi
$$

When $\varepsilon=0$, we find that the term diverges at $T=T_{c}$ without any assumption on the function $v$. Moreover, if the potential $U$ is a constant, then the solution to the BCS-Bogoliubov gap equation (1.1) depends on the temperature $T$ only, and does not depend on the energy $\xi$ (see Proposition 1.1). So the term (1.7) becomes

$$
-N_{0}\left\{\frac{\partial}{\partial T} u_{0}(T)^{2}\right\} \int_{\varepsilon}^{\hbar \omega_{D}} \frac{1}{\sqrt{\xi^{2}+u_{0}(T)^{2}}} d \xi .
$$

Note that $u_{0}\left(T_{c}\right)=0$ and that

$$
\left.\frac{\partial}{\partial T} u_{0}(T)^{2}\right|_{T=T_{c}}=-v .
$$

Here, $v$ is a constant, and it is assumed frequently that $v>0$ in the BCS-Bogoliubov model. The term (1.8) then becomes, at $T=T_{c}$,

$$
N_{0} v \int_{\varepsilon}^{\hbar \omega_{D}} \frac{1}{\xi} d \xi
$$

When $\varepsilon=0$, we again find that the term diverges at $T=T_{c}$. This is why we need to introduce $\varepsilon>0$ both in the BCS-Bogoliubov gap equation (1.1) and in the difference $\Psi$ given by (1.6).

\section{Main results}

Let the potential $U(\cdot, \cdot)$ satisfy the following:

$$
U(\cdot, \cdot) \in C\left(\left[\varepsilon, \hbar \omega_{D}\right]^{2}\right), \quad(0<) U_{1}<U(x, \xi)<U_{2} \quad \text { at all } \quad(x, \xi) \in\left[\varepsilon, \hbar \omega_{D}\right]^{2} .
$$


Then, by Theorem 1.6, there is a unique non-negative solution $u_{0}(T, \cdot) \in V_{T}$ to the BCSBogoliubov gap equation (1.1). By Definition 1.8, the transition temperature $T_{c}>0$ is thus defined. Note that the transition temperature $T_{c}>0$ is related to the solution $u_{0}(T, \cdot) \in V_{T}$.

The function

$$
(T, x) \mapsto \int_{\varepsilon}^{\hbar \omega_{D}} \frac{U(x, \xi)}{\sqrt{\xi^{2}+\Delta_{2}(T)^{2}}} \tanh \frac{\sqrt{\xi^{2}+\Delta_{2}(T)^{2}}}{2 T} d \xi
$$

is continuous and its value is less than one. This is because

$$
\begin{aligned}
& \int_{\varepsilon}^{\hbar \omega_{D}} \frac{U(x, \xi)}{\sqrt{\xi^{2}+\Delta_{2}(T)^{2}}} \tanh \frac{\sqrt{\xi^{2}+\Delta_{2}(T)^{2}}}{2 T} d \xi \\
& \quad<\int_{\varepsilon}^{\hbar \omega_{D}} \frac{U_{2}}{\sqrt{\xi^{2}+\Delta_{2}(T)^{2}}} \tanh \frac{\sqrt{\xi^{2}+\Delta_{2}(T)^{2}}}{2 T} d \xi \\
& \quad=1
\end{aligned}
$$

by (1.4). For example, if the potential $U(x, \xi)$ is nearly equal to $0.8 U_{2}$, then

$$
\int_{\varepsilon}^{\hbar \omega_{D}} \frac{U(x, \xi)}{\sqrt{\xi^{2}+\Delta_{2}(T)^{2}}} \tanh \frac{\sqrt{\xi^{2}+\Delta_{2}(T)^{2}}}{2 T} d \xi
$$

is nearly equal to 0.8 . Note that the function

$$
(T, x) \mapsto \int_{\varepsilon}^{\hbar \omega_{D}} \frac{U(x, \xi)}{\xi} \tanh \frac{\xi}{2 T} d \xi
$$

is also continuous.

We choose suitable $\tau>0$ and $\varepsilon>0$ such that $\tau<T_{c}$ and

$$
\int_{\varepsilon}^{\hbar \omega_{D}} \frac{U(x, \xi)}{\sqrt{\xi^{2}+\Delta_{2}(T)^{2}}} \tanh \frac{\sqrt{\xi^{2}+\Delta_{2}(T)^{2}}}{2 T} d \xi+\frac{\Delta_{2}(\tau)^{2}}{2 \varepsilon^{2}} \int_{\varepsilon}^{\hbar \omega_{D}} \frac{U(x, \xi)}{\xi} \tanh \frac{\xi}{2 T} d \xi<1 .
$$

The first term on the left-hand side of (2.2) is less that one as mentioned above. The second term tends to zero as $\Delta_{2}(\tau) / \varepsilon \rightarrow 0$ since

$$
\begin{aligned}
& \frac{\Delta_{2}(\tau)^{2}}{2 \varepsilon^{2}} \int_{\varepsilon}^{\hbar \omega_{D}} \frac{U(x, \xi)}{\xi} \tanh \frac{\xi}{2 T} d \xi \\
& <\frac{\Delta_{2}(\tau)^{2}}{2 \varepsilon^{2}} U_{2} \ln \frac{\hbar \omega_{D}}{\varepsilon} \\
& =\frac{\Delta_{2}(\tau)^{2}}{2 \varepsilon^{2}} U_{2} \ln \left\{\cosh \frac{1}{U_{2}}+\sqrt{1+\frac{\Delta_{2}(0)^{2}}{\varepsilon^{2}}} \sinh \frac{1}{U_{2}}\right\} \\
& \rightarrow 0 \text { as } \frac{\Delta_{2}(\tau)}{\varepsilon} \rightarrow 0 .
\end{aligned}
$$

Here we used the equality (see Proposition 1.1)

$$
\Delta_{2}(0)=\frac{\sqrt{\left(\hbar \omega_{D}-\varepsilon e^{1 / U_{2}}\right)\left(\hbar \omega_{D}-\varepsilon e^{-1 / U_{2}}\right)}}{\sinh \left(1 / U_{2}\right)} .
$$




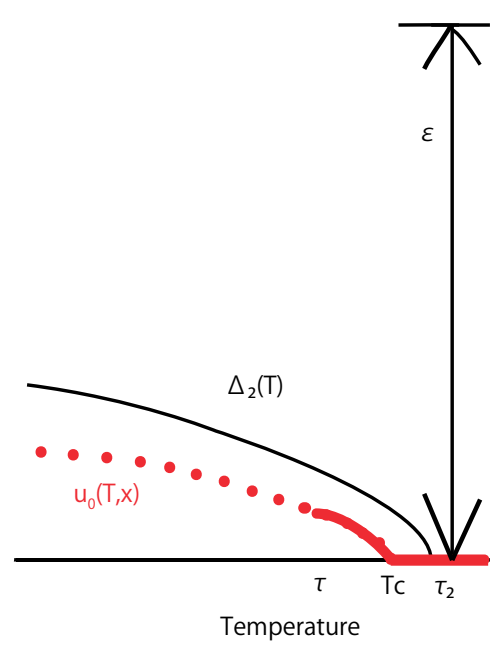

FIGURE 2. The graph of the solution $u_{0} \in \bar{W}$ with the energy $x$ fixed.

Remark 2.1. The function $\Delta_{2}(T)$ is strictly decreasing with respect to $T$ and tends to zero as $T \rightarrow \tau_{2}$, while $\varepsilon>0$ is fixed and is not equal to zero. Therefore, there is a certain $\tau>0$ satisfying $\Delta_{2}(T)<\varepsilon$ for $T \in\left[\tau, T_{c}\right]$. See Figure 2 . Hence $\Delta_{2}(\tau) / \varepsilon<1$. Thus we can choose suitable $\tau>0$ and $\varepsilon>0$ such that the inequality (2.2) holds true.

We then fix $\tau$ and $\varepsilon$ in (2.2), and we deal with the set $\left[\tau, T_{c}\right] \times\left[\varepsilon, \hbar \omega_{D}\right] \in \mathbb{R}^{2}$. Note that the left-hand side of (2.2) is a continuous function of $(T, x) \in\left[\tau, T_{c}\right] \times\left[\varepsilon, \hbar \omega_{D}\right]$. So we set

$$
\begin{aligned}
\alpha=\max _{(T, x) \in\left[\tau, T_{C}\right] \times\left[\varepsilon, \hbar \omega_{D}\right]}[ & \int_{\varepsilon}^{\hbar \omega_{D}} \frac{U(x, \xi)}{\sqrt{\xi^{2}+\Delta_{2}(T)^{2}}} \tanh \frac{\sqrt{\xi^{2}+\Delta_{2}(T)^{2}}}{2 T} d \xi \\
& \left.+\frac{\Delta_{2}(\tau)^{2}}{2 \varepsilon^{2}} \int_{\varepsilon}^{\hbar \omega_{D}} \frac{U(x, \xi)}{\xi} \tanh \frac{\xi}{2 T} d \xi\right] .
\end{aligned}
$$

Then

$$
\alpha<1
$$

We consider the following condition.

Condition $(C)$. Let $\tau$ and $\varepsilon$ be as above. An element $u \in C\left(\left[\tau, T_{C}\right] \times\left[\varepsilon, \hbar \omega_{D}\right]\right)$ is partially differentiable with respect to the temperature $T \in\left[\tau, T_{c}\right)$ twice, and both $(\partial u / \partial T)$ and $\left(\partial^{2} u / \partial T^{2}\right)$ belong to $C\left(\left[\tau, T_{c}\right) \times\left[\varepsilon, \hbar \omega_{D}\right]\right)$. Moreover, for the $u$ above, there are a unique $v \in C\left[\varepsilon, \hbar \omega_{D}\right]$ and a unique $w \in C\left[\varepsilon, \hbar \omega_{D}\right]$ satisfying the following.

(C1) $v(x)>0$ at all $x \in\left[\varepsilon, \hbar \omega_{D}\right]$.

(C2) For an arbitrary $\varepsilon_{1}>0$, there is a $\delta>0$ such that $\left|T_{c}-T\right|<\delta$ implies

$$
\left|v(x)-\frac{u(T, x)^{2}}{T_{c}-T}\right|<T_{c} \varepsilon_{1} \quad \text { and } \quad\left|v(x)+2 u(T, x) \frac{\partial u}{\partial T}(T, x)\right|<T_{c} \varepsilon_{1} .
$$

Here, $\delta$ does not depend on $x \in\left[\varepsilon, \hbar \omega_{D}\right]$. 
(C3) For an arbitrary $\varepsilon_{1}>0$, there is a $\delta>0$ such that $\left|T_{c}-T\right|<\delta$ implies

$$
\left|\frac{w(x)}{2}+\frac{u(T, x)^{2}+\left(T_{c}-T\right) \frac{\partial}{\partial T}\left\{u(T, x)^{2}\right\}}{\left(T_{c}-T\right)^{2}}\right|<\varepsilon_{1}
$$

and

$$
\left|w(x)-\frac{\partial^{2}}{\partial T^{2}}\left\{u(T, x)^{2}\right\}\right|<\varepsilon_{1} .
$$

Here, $\delta$ does not depend on $x \in\left[\varepsilon, \hbar \omega_{D}\right]$.

We then define our operator $A$ (see (1.2)) on the following subset $W$ of the Banach space $C\left(\left[\tau, T_{c}\right] \times\left[\varepsilon, \hbar \omega_{D}\right]\right):$

$$
A u(T, x)=\int_{\varepsilon}^{\hbar \omega_{D}} \frac{U(x, \xi) u(T, \xi)}{\sqrt{\xi^{2}+u(T, \xi)^{2}}} \tanh \frac{\sqrt{\xi^{2}+u(T, \xi)^{2}}}{2 T} d \xi, \quad u \in W
$$

where

$$
\begin{aligned}
W=\{u & \in C\left(\left[\tau, T_{c}\right] \times\left[\varepsilon, \hbar \omega_{D}\right]\right): u(T, x) \geq u\left(T^{\prime}, x\right)\left(T<T^{\prime}\right), \\
& \Delta_{1}(T) \leq u(T, x) \leq \Delta_{2}(T) \text { at }(T, x),\left(T^{\prime}, x\right) \in\left[\tau, T_{c}\right] \times\left[\varepsilon, \hbar \omega_{D}\right], \\
& u \text { satisfies Condition (C) above }\} .
\end{aligned}
$$

Remark 2.2. It follows directly from Condition (C2) that $u\left(T_{c}, x\right)=0$ at all $x \in\left[\varepsilon, \hbar \omega_{D}\right]$ for $u \in W$.

We denote by $\bar{W}$ the closure of the subset $W$ with respect to the norm $\|\cdot\|$ of the Banach space $C\left(\left[\tau, T_{c}\right] \times\left[\varepsilon, \hbar \omega_{D}\right]\right)$.

The following are our main results.

THEOREM 2.3. Let $U(\cdot, \cdot)$ satisfy (2.1). Choose $\tau>0$ and $\varepsilon>0$ such that (2.3) holds true. Then the operator $A: \bar{W} \rightarrow \bar{W}$ is a contraction operator, and hence there is a unique fixed point $u_{0} \in \bar{W}$ of the operator $A: \bar{W} \rightarrow \bar{W}$. Consequently, there is a unique non-negative solution $u_{0} \in \bar{W}$ to the BCS-Bogoliubov gap equation (1.1):

$$
\begin{gathered}
u_{0}(T, x)=\int_{\varepsilon}^{\hbar \omega_{D}} \frac{U(x, \xi) u_{0}(T, \xi)}{\sqrt{\xi^{2}+u_{0}(T, \xi)^{2}}} \tanh \frac{\sqrt{\xi^{2}+u_{0}(T, \xi)^{2}}}{2 T} d \xi, \\
(T, x) \in\left[\tau, T_{c}\right] \times\left[\varepsilon, \hbar \omega_{D}\right] .
\end{gathered}
$$

The solution $u_{0}$ is continuous on $\left[\tau, T_{c}\right] \times\left[\varepsilon, \hbar \omega_{D}\right]$, and is monotone decreasing with respect to the temperature $T$. Moreover, the solution $u_{0}$ satisfies that $\Delta_{1}(T) \leq u(T, x) \leq \Delta_{2}(T)$ at all $(T, x) \in\left[\tau, T_{c}\right] \times\left[\varepsilon, \hbar \omega_{D}\right]$ and that $u_{0}\left(T_{c}, x\right)=0$ at all $x \in\left[\varepsilon, \hbar \omega_{D}\right]$. If $u_{0} \in W$, then the solution $u_{0}$ satisfies Condition $(C)$. On the other hand, if $u_{0} \in \bar{W} \backslash W$, then the solution $u_{0}$ is approximated by an element of the subset $W$ fulfilling Condition $(C)$.

See Figure 2 for the graph of the solution $u_{0} \in \bar{W}$ with the energy $x$ fixed. Since $u_{0} \in \bar{W}$ by Theorem 2.3, we have $u_{0} \in W$ or $u_{0} \in \bar{W} \backslash W$. If $u_{0} \in \bar{W} \backslash W$, then the solution $u_{0}$ is approximated by a suitably chosen element $u_{1} \in W$, as mentioned in Theorem 2.3. In (1.6) we then replace the solution $u_{0} \in \bar{W} \backslash W$ by this element $u_{1} \in W$. Once we replace the solution $u_{0} \in \bar{W} \backslash W$ of (1.6) by this $u_{1} \in W$, we see that all the conditions of Definition 1.10 are satisfied. We immediately have the following result. 
THEOREM 2.4

(1) Suppose that $u_{0} \in W$. Then all the conditions of Definition 1.10 are satisfied. Consequently the transition from a normal conducting state to a superconducting state at $T=T_{c}$ is a second-order phase transition.

(2) Suppose that $u_{0} \in \bar{W} \backslash W$. In (1.6), we replace $u_{0} \in \bar{W} \backslash W$ by a suitably chosen element $u_{1} \in W$. Then all the conditions of Definition 1.10 are satisfied. Consequently the transition from a normal conducting state to a superconducting state at $T=T_{c}$ is a second-order phase transition.

Let $g:[0, \infty) \rightarrow \mathbb{R}$ be given by

$$
g(\eta)= \begin{cases}\frac{1}{\eta^{2} \cosh ^{2} \eta}-\frac{\tanh \eta}{\eta^{3}} & (\eta>0), \\ -\frac{2}{3} & (\eta=0) .\end{cases}
$$

Note that $g(\eta)<0$. See Lemma 4.4 for some properties of the function $g$.

We recall here that the gap $\Delta C_{V}$ in the specific heat at constant volume at $T=T_{c}$ is given by Remark 1.11. The following gives the exact and explicit expression for the gap.

Proposition 2.5. Let $v$ be as in (C2) of Condition (C), and $g$ as above. Then the gap $\Delta C_{V}$ in the specific heat at constant volume at $T=T_{c}$ is given by

$$
\Delta C_{V}=-\frac{N_{0}}{8 T_{c}} \int_{\varepsilon /\left(2 T_{c}\right)}^{\hbar \omega_{D} /\left(2 T_{c}\right)} v\left(2 T_{c} \eta\right)^{2} g(\eta) d \eta \quad(>0) .
$$

\section{Proof of Theorem 2.3}

In this section we give a proof of Theorem 2.3. We first show that $A: W \rightarrow W$.

Lemma 3.1. If $u \in W$, then $A u \in C\left(\left[\tau, T_{c}\right] \times\left[\varepsilon, \hbar \omega_{D}\right]\right)$.

Proof. Let $(T, x),\left(T_{1}, x_{1}\right) \in\left[\tau, T_{c}\right] \times\left[\varepsilon, \hbar \omega_{D}\right]$. For $u \in W$,

$$
A u(T, x)-A u\left(T_{1}, x_{1}\right)=A u(T, x)-A u\left(T, x_{1}\right)+A u\left(T, x_{1}\right)-A u\left(T_{1}, x_{1}\right) .
$$

By $(2.1)$ the potential $U(\cdot, \cdot)$ is uniformly continuous on $\left[\varepsilon, \hbar \omega_{D}\right]^{2}$, and hence for an arbitrary $\varepsilon_{1}>0$, there is a $\delta_{1}>0$ such that $\left|x-x_{1}\right|<\delta_{1}$ implies

$$
\left|U(x, \xi)-U\left(x_{1}, \xi\right)\right|<\frac{\varepsilon_{1}}{3 \hbar \omega_{D}} \quad \text { at all } \xi \in\left[\varepsilon, \hbar \omega_{D}\right] .
$$

Note that $\delta_{1}$ does not depend on $x$, nor on $x_{1}$, nor on $\xi$, nor on $T$, nor on $T_{1}$. The first and second terms on the right-hand side of (3.1) therefore turn into

$$
\begin{aligned}
\left|A u(T, x)-A u\left(T, x_{1}\right)\right| & \leq \int_{\varepsilon}^{\hbar \omega_{D}} \frac{\left|U(x, \xi)-U\left(x_{1}, \xi\right)\right| u(T, \xi)}{\sqrt{\xi^{2}+u(T, \xi)^{2}}} \tanh \frac{\sqrt{\xi^{2}+u(T, \xi)^{2}}}{2 T} d \xi \\
& \leq \int_{\varepsilon}^{\hbar \omega_{D}}\left|U(x, \xi)-U\left(x_{1}, \xi\right)\right| d \xi \\
& <\frac{\varepsilon_{1}}{3} .
\end{aligned}
$$


On the other hand, the third and fourth terms become

$$
A u\left(T, x_{1}\right)-A u\left(T_{1}, x_{1}\right)=\int_{\varepsilon}^{\hbar \omega_{D}} U\left(x_{1}, \xi\right)\left\{K_{1}+K_{2}\right\} d \xi
$$

where

$$
\begin{aligned}
K_{1}= & \frac{u(T, \xi)}{\sqrt{\xi^{2}+u(T, \xi)^{2}}} \tanh \frac{\sqrt{\xi^{2}+u(T, \xi)^{2}}}{2 T} \\
& -\frac{u\left(T_{1}, \xi\right)}{\sqrt{\xi^{2}+u\left(T_{1}, \xi\right)^{2}}} \tanh \frac{\sqrt{\xi^{2}+u\left(T_{1}, \xi\right)^{2}}}{2 T}, \\
K_{2}= & \frac{u\left(T_{1}, \xi\right)}{\sqrt{\xi^{2}+u\left(T_{1}, \xi\right)^{2}}}\left\{\tanh \frac{\sqrt{\xi^{2}+u\left(T_{1}, \xi\right)^{2}}}{2 T}-\tanh \frac{\sqrt{\xi^{2}+u\left(T_{1}, \xi\right)^{2}}}{2 T_{1}}\right\} .
\end{aligned}
$$

Note that $u \in W$ is uniformly continuous on $\left[\tau, T_{c}\right] \times\left[\varepsilon, \hbar \omega_{D}\right]$. Then, for the $\varepsilon_{1}>0$ above, there is a $\delta_{2}>0$ such that $\left|T-T_{1}\right|<\delta_{2}$ implies

$$
\left|u(T, \xi)-u\left(T_{1}, \xi\right)\right|<\frac{\varepsilon_{1}}{3 \alpha} .
$$

Here, $\alpha$ is that in (2.3), and $\delta_{2}$ does not depend on $x$, nor on $x_{1}$, nor on $\xi$, nor on $T$, nor on $T_{1}$. However, $\delta_{2}$ may depend on $u \in W$. Note that $z / \cosh ^{2} z \leq \tanh z(z \geq 0)$. Hence

$$
\begin{array}{rl}
\int_{\varepsilon}^{\hbar \omega_{D}} & U\left(x_{1}, \xi\right)\left|K_{1}\right| d \xi \\
= & \int_{\varepsilon}^{\hbar \omega_{D}} \frac{U\left(x_{1}, \xi\right)}{\left(\xi^{2}+c^{2}\right)^{3 / 2}}\left\{\xi^{2} \tanh \frac{\sqrt{\xi^{2}+c^{2}}}{2 T}+c^{2} \frac{\sqrt{\xi^{2}+c^{2}}}{2 T} \frac{1}{\cosh ^{2}\left(\sqrt{\xi^{2}+c^{2}} / 2 T\right)}\right\} \\
& \times\left|u(T, \xi)-u\left(T_{1}, \xi\right)\right| d \xi \\
\leq & \int_{\varepsilon}^{\hbar \omega_{D}} \frac{U\left(x_{1}, \xi\right)}{\sqrt{\xi^{2}+c^{2}}} \tanh \frac{\sqrt{\xi^{2}+c^{2}}}{2 T}\left|u(T, \xi)-u\left(T_{1}, \xi\right)\right| d \xi .
\end{array}
$$

Here, $c$ is between $u(T, \xi)$ and $u\left(T_{1}, \xi\right)$. Note again that $z / \cosh ^{2} z \leq \tanh z(z \geq 0)$ and that the function $z \mapsto(\tanh z) / z(z \geq 0)$ is strictly decreasing. Then a straightforward calculation gives

$$
\begin{aligned}
\frac{1}{\sqrt{\xi^{2}+c^{2}}} \tanh \frac{\sqrt{\xi^{2}+c^{2}}}{2 T} & \\
= & \frac{1}{\sqrt{\xi^{2}+\Delta_{2}(T)^{2}}} \tanh \frac{\sqrt{\xi^{2}+\Delta_{2}(T)^{2}}}{2 T} \\
& +\frac{\Delta_{2}(T)^{2}-c^{2}}{2\left(\xi^{2}+c_{1}^{2}\right)^{3 / 2}}\left\{\tanh \frac{\sqrt{\xi^{2}+c_{1}^{2}}}{2 T}-\frac{\sqrt{\xi^{2}+c_{1}^{2}}}{2 T} \frac{\cosh ^{2}\left(\sqrt{\xi^{2}+c_{1}^{2}} / 2 T\right)}{2 T}\right\} \\
\leq & \frac{1}{\sqrt{\xi^{2}+\Delta_{2}(T)^{2}}} \tanh \frac{\sqrt{\xi^{2}+\Delta_{2}(T)^{2}}}{2 T}+\frac{\Delta_{2}(\tau)^{2}}{2 \varepsilon^{2}} \frac{1}{\xi} \tanh \frac{\xi}{2 T},
\end{aligned}
$$


where $c_{1}$ satisfies $c<c_{1}<\Delta_{2}(T)$. Hence

$$
\int_{\varepsilon}^{\hbar \omega_{D}} U\left(x_{1}, \xi\right)\left|K_{1}\right| d \xi<\frac{\varepsilon_{1}}{3 \alpha} \alpha=\frac{\varepsilon_{1}}{3}
$$

Moreover, if

$$
\left|T-T_{1}\right|<\frac{2 \tau^{2} \varepsilon_{1}}{3 U_{2} \Delta_{2}(\tau) \hbar \omega_{D}}
$$

then

$$
\begin{aligned}
\int_{\varepsilon}^{\hbar \omega_{D}} U\left(x_{1}, \xi\right)\left|K_{2}\right| d \xi & \leq U_{2} \int_{\varepsilon}^{\hbar \omega_{D}} \frac{u\left(T_{1}, \xi\right)}{2\left(T^{\prime \prime}\right)^{2} \cosh ^{2}\left(\sqrt{\xi^{2}+u\left(T_{1}, \xi\right)^{2}} / 2 T^{\prime \prime}\right)} d \xi\left|T-T_{1}\right| \\
& \leq U_{2} \int_{\varepsilon}^{\hbar \omega_{D}} \frac{\Delta_{2}(\tau)}{2 \tau^{2}} d \xi\left|T-T_{1}\right| \\
& <\frac{\varepsilon_{1}}{3} .
\end{aligned}
$$

Here, $T^{\prime \prime}$ is between $T$ and $T_{1}$. Thus

$$
\left|A u(T, x)-A u\left(T_{1}, x_{1}\right)\right|<\varepsilon_{1},
$$

where

$$
\left|T-T_{1}\right|+\left|x-x_{1}\right|<\delta=\min \left(\delta_{1}, \delta_{2}, \frac{2 \tau^{2} \varepsilon_{1}}{3 U_{2} \Delta_{2}(\tau) \hbar \omega_{D}}\right) .
$$

Lemma 3.2. Let $(T, x),\left(T_{1}, x\right) \in\left[\tau, T_{c}\right] \times\left[\varepsilon, \hbar \omega_{D}\right]$, and let $T<T_{1}$. If $u \in W$, then $A u(T, x) \geq A u\left(T_{1}, x\right)$.

Proof. Since $T<T_{1}$,

$$
\frac{u(T, \xi)}{\sqrt{\xi^{2}+u(T, \xi)^{2}}} \geq \frac{u\left(T_{1}, \xi\right)}{\sqrt{\xi^{2}+u\left(T_{1}, \xi\right)^{2}}} .
$$

Hence, $K_{1} \geq 0$ and $K_{2} \geq 0$ in (3.2). Thus

$$
A u(T, x)-A u\left(T_{1}, x\right) \geq 0 .
$$

Lemma 3.3. Let $(T, x) \in\left[\tau, T_{c}\right] \times\left[\varepsilon, \hbar \omega_{D}\right]$. If $u \in W$, then $\Delta_{1}(T) \leq A u(T, x) \leq \Delta_{2}(T)$.

Proof. Since

$$
\frac{u(T, \xi)}{\sqrt{\xi^{2}+u(T, \xi)^{2}}} \leq \frac{\Delta_{2}(T)}{\sqrt{\xi^{2}+\Delta_{2}(T)^{2}}}
$$

it follows from (1.4) that

$$
A u(T, x) \leq U_{2} \int_{\varepsilon}^{\hbar \omega_{D}} \frac{\Delta_{2}(T)}{\sqrt{\xi^{2}+\Delta_{2}(T)^{2}}} \tanh \frac{\sqrt{\xi^{2}+\Delta_{2}(T)^{2}}}{2 T} d \xi=\Delta_{2}(T) .
$$

Similarly we can show that $\Delta_{1}(T) \leq A u(T, x)$. 
We now show that $A u(u \in W)$ satisfies Condition (C) so as to conclude that $A: W \rightarrow W$. A straightforward calculation gives the following.

Lemma 3.4. Let $u \in W$. Then $A u$ is partially differentiable with respect to $T \in\left[\tau, T_{c}\right.$ ) twice, and

$$
\frac{\partial A u}{\partial T}, \frac{\partial^{2} A u}{\partial T^{2}} \in C\left(\left[\tau, T_{C}\right) \times\left[\varepsilon, \hbar \omega_{D}\right]\right) .
$$

For $u \in W$, let $v$ be as in Condition (C). Note that $v$ depends on $u$. Set

$$
F(x)=\left\{\int_{\varepsilon}^{\hbar \omega_{D}} U(x, \xi) \frac{\sqrt{v(\xi)}}{\xi} \tanh \frac{\xi}{2 T_{C}} d \xi\right\}^{2} \quad(>0), \quad \varepsilon \leq x \leq \hbar \omega_{D} .
$$

Lemma 3.5. Suppose $u \in W$. Then the function $F$ given by (3.4) belongs to $C\left[\varepsilon, \hbar \omega_{D}\right]$, and for an arbitrary $\varepsilon_{1}>0$, there is a $\delta>0$ such that $\left|T_{c}-T\right|<\delta$ implies

$$
\left|F(x)-\frac{\{A u(T, x)\}^{2}}{T_{c}-T}\right|<T_{c} \varepsilon_{1} \quad \text { and }\left|F(x)+2 A u(T, x) \frac{\partial A u}{\partial T}(T, x)\right|<T_{c} \varepsilon_{1} .
$$

Here, $\delta$ does not depend on $x \in\left[\varepsilon, \hbar \omega_{D}\right]$. Such a function $F$ is uniquely given by (3.4).

Proof. Since the potential $U(\cdot, \cdot)$ is uniformly continuous on $\left[\varepsilon, \hbar \omega_{D}\right]^{2}$ by $(2.1)$, the function $F$ is continuous on $\left[\varepsilon, \hbar \omega_{D}\right]$. Moreover,

$$
\left|F(x)-\frac{\{A u(T, x)\}^{2}}{T_{c}-T}\right| \leq U_{2}^{2} I_{0}\left(I_{1}+I_{2}+I_{3}\right),
$$

where

$$
\begin{aligned}
& I_{0}=\int_{\varepsilon}^{\hbar \omega_{D}} \frac{1}{\xi}\left(\sqrt{v(\xi)}+\sqrt{\frac{u(T, \xi)^{2}}{T_{c}-T}}\right) d \xi \\
& I_{1}=\int_{\varepsilon}^{\hbar \omega_{D}} \frac{1}{\xi} \mid \sqrt{v(\xi)}-\sqrt{\frac{u(T, \xi)^{2}}{T_{c}-T} \mid \tanh \frac{\xi}{2 T_{c}} d \xi,} \\
& I_{2}=\int_{\varepsilon}^{\hbar \omega_{D}} \sqrt{\frac{u(T, \xi)^{2}}{T_{c}-T}}\left|\frac{1}{\xi}-\frac{1}{\sqrt{\xi^{2}+u(T, \xi)^{2}}}\right| \tanh \frac{\xi}{2 T_{c}} d \xi, \\
& I_{3}=\int_{\varepsilon}^{\hbar \omega_{D}} \sqrt{\frac{u(T, \xi)^{2}}{T_{c}-T}} \frac{1}{\sqrt{\xi^{2}+u(T, \xi)^{2}}}\left|\tanh \frac{\xi}{2 T_{c}}-\tanh \frac{\sqrt{\xi^{2}+u(T, \xi)^{2}}}{2 T}\right| d \xi .
\end{aligned}
$$

By Condition (C2), for $(0<) \varepsilon_{1}<1$, there is a $\delta_{1}>0$ such that $T_{c}-T<\delta_{1}$ implies

$$
\frac{u(T, \xi)^{2}}{T_{c}-T}<v(\xi)+T_{c} \varepsilon_{1}<v(\xi)+T_{c} .
$$

Note that $\delta_{1}$ does not depend on $x$, nor on $\xi$. Moreover, for $(0<) \varepsilon_{1}<1$, there is a $\delta_{2}>0$ such that $T_{c}-T<\delta_{2}$ implies

$$
u(T, \xi)^{2}=\frac{u(T, \xi)^{2}}{T_{c}-T}\left(T_{c}-T\right)<\left\{\max _{\xi \in\left[\varepsilon, \hbar \omega_{D}\right]} v(\xi)+T_{c}\right\}\left(T_{c}-T\right)<T_{c}^{2} \varepsilon_{1}^{2} .
$$

Here,

$$
\delta_{2}=\frac{T_{c}^{2} \varepsilon_{1}^{2}}{\max _{\xi \in\left[\varepsilon, \hbar \omega_{D}\right]} v(\xi)+T_{c}}
$$

and $\delta_{2}$ does not depend on $x$, nor on $\xi$. 
Noting that $(\tanh z) / z \leq 1(z \geq 0)$, we find

$$
\begin{aligned}
I_{0} & <2 \sqrt{\max _{\xi \in\left[\varepsilon, \hbar \omega_{D}\right]} v(\xi)+T_{c}} \ln \frac{\hbar \omega_{D}}{\varepsilon}, \\
I_{1} & <\frac{\varepsilon}{2} \int_{\varepsilon}^{\hbar \omega_{D}} \frac{d \xi}{\sqrt{v(\xi)}}, \\
I_{2} & \leq \sqrt{\max _{\xi \in\left[\varepsilon, \hbar \omega_{D}\right]} v(\xi)+T_{c}} \int_{\varepsilon}^{\hbar \omega_{D}} \frac{u(T, \xi)}{2 T_{c} \xi} d \xi<\sqrt{\max _{\xi \in\left[\varepsilon, \hbar \omega_{D}\right]} v(\xi)+T_{c}} \frac{\varepsilon}{2} \ln \frac{\hbar \omega_{D}}{\varepsilon}, \\
I_{3} & \leq \sqrt{\max _{\xi \in\left[\varepsilon, \hbar \omega_{D}\right]} v(\xi)+T_{c}} \int_{\varepsilon}^{\hbar \omega_{D}}\left\{\frac{u(T, \xi)}{4 \tau \xi}+\frac{T_{c}-T}{2 \tau^{2}}\right\} d \xi \\
& <\sqrt{\max _{\xi \in\left[\varepsilon, \hbar \omega_{D}\right]} v(\xi)+T_{c}}\left(\frac{1}{4 \tau} \ln \frac{\hbar \omega_{D}}{\varepsilon}+\frac{\hbar \omega_{D}}{2 \tau^{2}}\right) T_{c} \varepsilon_{1} .
\end{aligned}
$$

Here, $T_{c}-T<\delta=\min \left(\delta_{1}, \delta_{2}, T_{c} \varepsilon_{1}\right)$. Note that $\delta$ does not depend on $x \in\left[\varepsilon, \hbar \omega_{D}\right]$. Uniqueness of $F$ follows immediately.

We can show that

$$
\left|F(x)+2 A u(T, x) \frac{\partial A u}{\partial T}(T, x)\right|<T_{c} \varepsilon_{1}
$$

similarly.

For $u \in W$, let $v$ and $w$ be as in Condition (C). Note that $v$ and $w$ depend on $u$. Set

$$
\begin{aligned}
G(x)= & \int_{\varepsilon}^{\hbar \omega_{D}} U(x, \xi) \frac{\sqrt{v(\xi)}}{\xi} \tanh \frac{\xi}{2 T_{c}} d \xi \\
& \times \int_{\varepsilon}^{\hbar \omega_{D}} U(x, \eta)\left\{\left(\frac{w(\eta)}{\eta \sqrt{v(\eta)}}-\frac{2 \sqrt{v(\eta)^{3}}}{\eta^{3}}\right) \tanh \frac{\eta}{2 T_{c}}\right. \\
& \left.+\frac{\sqrt{v(\eta)}}{\cosh ^{2}\left(\eta / 2 T_{c}\right)}\left(\frac{v(\eta)}{\eta^{2} T_{c}}+\frac{2}{T_{c}^{2}}\right)\right\} d \eta
\end{aligned}
$$

where $\varepsilon \leq x \leq \hbar \omega_{D}$.

Lemma 3.6. Suppose $u \in W$. Then the function $G$ given by (3.5) belongs to $C\left[\varepsilon, \hbar \omega_{D}\right]$, and for an arbitrary $\varepsilon_{1}>0$, there is a $\delta>0$ such that $\left|T_{c}-T\right|<\delta$ implies

$$
\left|\frac{G(x)}{2}+\frac{\{A u(T, x)\}^{2}+\left(T_{c}-T\right) \frac{\partial}{\partial T}\left\{A u(T, x)^{2}\right\}}{\left(T_{c}-T\right)^{2}}\right|<\varepsilon_{1}
$$

and

$$
\left|G(x)-\frac{\partial^{2}}{\partial T^{2}}\left\{A u(T, x)^{2}\right\}\right|<\varepsilon_{1} .
$$

Here, $\delta$ does not depend on $x \in\left[\varepsilon, \hbar \omega_{D}\right]$. Such a function $G$ is uniquely given by (3.5).

Proof. Since the potential $U(\cdot, \cdot)$ is uniformly continuous on $\left[\varepsilon, \hbar \omega_{D}\right]^{2}$ by $(2.1)$, the function $G$ is continuous on $\left[\varepsilon, \hbar \omega_{D}\right]$. A discussion similar to that in the proof of Lemma 3.5 shows the rest. This time we also need Condition (C3) in Section 2. 
The lemmas above immediately give the following result.

LeMma 3.7. $A: W \rightarrow W$.

As mentioned above, we denote by $\|\cdot\|$ the norm of the Banach space $C\left(\left[\tau, T_{c}\right] \times\right.$ $\left.\left[\varepsilon, \hbar \omega_{D}\right]\right)$.

Lemma 3.8. Let $\alpha$ be as in (2.3). Then $\|A u-A v\| \leq \alpha\|u-v\|$ for $u, v \in W$.

Proof. Let $u, v \in W$. Let $c$ be between $u(T, \xi)$ and $v(T, \xi)$. Since $z / \cosh ^{2} z \leq \tanh z(z \geq 0)$, it then follows that

$$
\begin{aligned}
|A u(T, x)-A v(T, x)| & \\
\leq & \int_{\varepsilon}^{\hbar \omega_{D}} U(x, \xi) \mid \frac{u(T, \xi)}{\sqrt{\xi^{2}+u(T, \xi)^{2}}} \tanh \frac{\sqrt{\xi^{2}+u(T, \xi)^{2}}}{2 T} \\
& -\frac{v(T, \xi)}{\sqrt{\xi^{2}+v(T, \xi)^{2}}} \tanh \frac{\sqrt{\xi^{2}+v(T, \xi)^{2}}}{2 T} \mid d \xi \\
\leq & \int_{\varepsilon}^{\hbar \omega_{D}} \frac{U(x, \xi)}{\left(\xi^{2}+c^{2}\right)^{3 / 2}}\left\{\xi^{2} \tanh \frac{\sqrt{\xi^{2}+c^{2}}}{2 T}\right. \\
& \left.+c^{2} \frac{\sqrt{\xi^{2}+c^{2}}}{2 T} \frac{\cosh ^{2}\left(\sqrt{\xi^{2}+c^{2}} / 2 T\right)}{2 T}\right\} d \xi\|u-v\| \\
\leq & \int_{\varepsilon}^{\hbar \omega_{D}} \frac{U(x, \xi)}{\sqrt{\xi^{2}+c^{2}}} \tanh ^{\frac{\sqrt{\xi^{2}+c^{2}}}{2 T}} d \xi\|u-v\| .
\end{aligned}
$$

A discussion similar to that in (3.3) gives

$$
\begin{aligned}
|A u(T, x)-A v(T, x)| \leq & \int_{\varepsilon}^{\hbar \omega_{D}} U(x, \xi)\left\{\frac{1}{\sqrt{\xi^{2}+\Delta_{2}(T)^{2}}} \tanh \frac{\sqrt{\xi^{2}+\Delta_{2}(T)^{2}}}{2 T}\right. \\
& \left.+\frac{\Delta_{2}(\tau)^{2}}{2 \varepsilon^{2}} \frac{1}{\xi} \tanh \frac{\xi}{2 T}\right\} d \xi\|u-v\| \\
\leq & \alpha\|u-v\| .
\end{aligned}
$$

We extend the domain $W$ of our operator $A$ to its closure $\bar{W}$. Let $u \in \bar{W}$. Then there is a sequence $\left\{u_{n}\right\}_{n=1}^{\infty} \subset W$ satisfying $\left\|u-u_{n}\right\| \rightarrow 0$ as $n \rightarrow \infty$. Lemma 3.8 gives that $\left\{A u_{n}\right\}_{n=1}^{\infty} \subset W$ is a Cauchy sequence, and hence there is an $A u \in \bar{W}$ satisfying $\| A u-$ $A u_{n} \| \rightarrow 0$ as $n \rightarrow \infty$. Note that $A u \in \bar{W}$ does not depend on the sequence $\left\{u_{n}\right\}_{n=1}^{\infty} \subset W$. We thus have the following lemma.

LeMma 3.9. $A: \bar{W} \rightarrow \bar{W}$.

It is not obvious that $A u(u \in \bar{W})$ is expressed as that in (1.2). The next lemma shows this is the case. 
Lemma 3.10. Let $u \in \bar{W}$. Then

$$
\begin{gathered}
A u(T, x)=\int_{\varepsilon}^{\hbar \omega_{D}} \frac{U(x, \xi) u(T, \xi)}{\sqrt{\xi^{2}+u(T, \xi)^{2}}} \tanh \frac{\sqrt{\xi^{2}+u(T, \xi)^{2}}}{2 T} d \xi, \\
(T, x) \in\left[\tau, T_{c}\right] \times\left[\varepsilon, \hbar \omega_{D}\right] .
\end{gathered}
$$

Proof. For $u \in \bar{W}$, set

$$
I(T, x)=\int_{\varepsilon}^{\hbar \omega_{D}} \frac{U(x, \xi) u(T, \xi)}{\sqrt{\xi^{2}+u(T, \xi)^{2}}} \tanh \frac{\sqrt{\xi^{2}+u(T, \xi)^{2}}}{2 T} d \xi
$$

and let $\left\{u_{n}\right\}_{n=1}^{\infty} \subset W$ be a sequence satisfying $\left\|u-u_{n}\right\| \rightarrow 0$ as $n \rightarrow \infty$. Note that the function $(T, x) \mapsto I(T, x)$ is well defined and continuous. Then

$$
|A u(T, x)-I(T, x)| \leq\left|A u(T, x)-A u_{n}(T, x)\right|+\left|A u_{n}(T, x)-I(T, x)\right| .
$$

Since $\left\|A u-A u_{n}\right\| \rightarrow 0$ as $n \rightarrow \infty$, the first term on the right-hand side becomes

$$
\left|A u(T, x)-A u_{n}(T, x)\right| \leq\left\|A u-A u_{n}\right\| \rightarrow 0 \quad(n \rightarrow \infty) .
$$

A discussion similar to that in the proof of Lemma 3.8 gives that the second term becomes

$$
\left|A u_{n}(T, x)-I(T, x)\right| \leq \alpha\left\|u_{n}-u\right\| \rightarrow 0 \quad(n \rightarrow \infty) .
$$

The result thus follows.

Lemma 3.8 immediately gives the following.

Lemma 3.11. Let $\alpha$ be as in (2.3). Then $\|A u-A v\| \leq a\|u-v\|$ for $u, v \in \bar{W}$. Consequently, the operator $A: \bar{W} \rightarrow \bar{W}$ is a contraction operator.

The Banach fixed point theorem thus implies the following lemma.

Lemma 3.12. The operator $A: \bar{W} \rightarrow \bar{W}$ has a unique fixed point $u_{0} \in \bar{W}$. Consequently, there is a unique non-negative solution $u_{0} \in \bar{W}$ to the BCS-Bogoliubov gap equation (1.1):

$$
\begin{gathered}
u_{0}(T, x)=\int_{\varepsilon}^{\hbar \omega_{D}} \frac{U(x, \xi) u_{0}(T, \xi)}{\sqrt{\xi^{2}+u_{0}(T, \xi)^{2}}} \tanh \frac{\sqrt{\xi^{2}+u_{0}(T, \xi)^{2}}}{2 T} d \xi \\
(T, x) \in\left[\tau, T_{c}\right] \times\left[\varepsilon, \hbar \omega_{D}\right] .
\end{gathered}
$$

Now our proof of Theorem 2.3 is complete.

\section{Proofs of Theorem 2.4 and Proposition 2.5}

We begin this section by preparing a lemma. As mentioned in Theorem 2.4, the function $u_{0}$ in (1.6) is the solution $u_{0} \in \bar{W}$ of Theorem 2.3; however, if $u_{0} \in \bar{W} \backslash W$, we then approximate $u_{0} \in \bar{W} \backslash W$ by a suitably chosen element $u_{1} \in W$ and we replace $u_{0}$ in (1.6) by this $u_{1} \in W$. 
We denote by $\Psi_{1}(T)$ the thermodynamic potential corresponding to this element $u_{1} \in W$ :

$$
\begin{aligned}
\Psi_{1}(T)= & -2 N_{0} \int_{\varepsilon}^{\hbar \omega_{D}}\left\{\sqrt{\xi^{2}+u_{1}(T, \xi)^{2}}-\xi\right\} d \xi \\
& +N_{0} \int_{\varepsilon}^{\hbar \omega_{D}} \frac{u_{1}(T, \xi)^{2}}{\sqrt{\xi^{2}+u_{1}(T, \xi)^{2}}} \tanh \frac{\sqrt{\xi^{2}+u_{1}(T, \xi)^{2}}}{2 T} d \xi \\
& -4 N_{0} T \int_{\varepsilon}^{\hbar \omega_{D}} \ln \frac{1+e^{-\sqrt{\xi^{2}+u_{1}(T, \xi)^{2}} / T}}{1+e^{-\xi / T}} d \xi, \quad T \in\left[\tau, T_{C}\right] .
\end{aligned}
$$

A discussion similar to that in the proof of Lemma 3.8 gives the following, which shows that $\Psi$ is approximated by $\Psi_{1}$.

LeMmA 4.1. Let $\Psi$ be as in (1.6) and $\Psi_{1}$ as in (4.1). Then, at all $T \in\left[\tau, T_{c}\right]$,

$$
\left|\Psi(T)-\Psi_{1}(T)\right| \leq 2 N_{0} \Delta_{2}(0)\left\{\left(1+2 \frac{T_{c}}{\tau}\right) \ln \frac{\hbar \omega_{D}}{\varepsilon}+\alpha\right\}\left\|u-u_{0}\right\|,
$$

where $\alpha$ is that in (2.3).

Remark 4.2. In what follows, when the solution $u_{0}$ to the BCS-Bogoliubov gap equation (1.1) is an element of $W$, we denote by $u$ below the very solution $u_{0} \in W$; when the solution $u_{0}$ is an element of $\bar{W} \backslash W$, we denote by $u$ below the suitably chosen element $u_{1} \in W$ mentioned just above. Therefore, in what follows, the function $u$ does not always denote the solution and is an element of $W$.

Lemma 4.3. Let $\Psi$ be as in (1.6). Then $\Psi$ is differentiable on $\left[\tau, T_{c}\right]$, and

$$
\Psi\left(T_{c}\right)=0 \quad \text { and } \quad \frac{\partial \Psi}{\partial T}\left(T_{c}\right)=0 .
$$

Proof. Note that $u \in W$, as mentioned in Remark 4.2. It then follows that $u\left(T_{c}, \xi\right)=0$ at all $\xi \in\left[\varepsilon, \hbar \omega_{D}\right]$ (see Remark 2.2 above). Hence $\Psi\left(T_{c}\right)=0$. A straightforward calculation gives that $\Psi$ is differentiable on $\left[\tau, T_{c}\right)$. So it suffices to show that $\Psi$ is differentiable at $T=T_{c}$ and that $(\partial \Psi / \partial T)\left(T_{c}\right)=0$. Note that $\Psi\left(T_{c}\right)=0$. Then

$$
\begin{aligned}
\frac{\Psi\left(T_{c}\right)-\Psi(T)}{T_{c}-T}= & 2 N_{0} \int_{\varepsilon}^{\hbar \omega_{D}} \frac{u(T, \xi)^{2}}{T_{c}-T} \frac{1}{\sqrt{\xi^{2}+u(T, \xi)^{2}}+\xi} d \xi \\
& -N_{0} \int_{\varepsilon}^{\hbar \omega_{D}} \frac{u(T, \xi)^{2}}{T_{c}-T} \frac{1}{\sqrt{\xi^{2}+u(T, \xi)^{2}}} \tanh \frac{\sqrt{\xi^{2}+u(T, \xi)^{2}}}{2 T} d \xi \\
& +4 N_{0} T \int_{\varepsilon}^{\hbar \omega_{D}} \frac{1}{T_{c}-T} \ln \frac{1+e^{-\sqrt{\xi^{2}+u(T, \xi)^{2}} / T}}{1+e^{-\xi / T}} d \xi
\end{aligned}
$$

By $(\mathrm{C} 2)$ of Condition $(\mathrm{C})$, for an arbitrary $(0<) \varepsilon_{1}<1$, there is a $\delta>0$ such that $\left|T_{c}-T\right|<\delta$ implies

$$
\frac{u(T, \xi)^{2}}{T_{c}-T} \frac{1}{\sqrt{\xi^{2}+u(T, \xi)^{2}}+\xi}<\frac{v(\xi)+T_{c} \varepsilon_{1}}{\xi}<\frac{v(\xi)+T_{c}}{\xi} .
$$


The Lebesgue dominated convergence theorem therefore implies that the first term on the right-hand side of (4.2) becomes

$$
2 N_{0} \lim _{T \uparrow T_{c}} \int_{\varepsilon}^{\hbar \omega_{D}} \frac{u(T, \xi)^{2}}{T_{c}-T} \frac{1}{\sqrt{\xi^{2}+u(T, \xi)^{2}}+\xi} d \xi=N_{0} \int_{\varepsilon}^{\hbar \omega_{D}} \frac{v(\xi)}{\xi} d \xi .
$$

We can deal with the second and third terms similarly. We get

$$
\begin{aligned}
- & N_{0} \lim _{T \uparrow T_{c}} \int_{\varepsilon}^{\hbar \omega_{D}} \frac{u(T, \xi)^{2}}{T_{c}-T} \frac{1}{\sqrt{\xi^{2}+u(T, \xi)^{2}}} \tanh \frac{\sqrt{\xi^{2}+u(T, \xi)^{2}}}{2 T} d \xi \\
& =-N_{0} \int_{\varepsilon}^{\hbar \omega_{D}} \frac{v(\xi)}{\xi} \tanh \frac{\xi}{2 T_{c}} d \xi
\end{aligned}
$$

and

$$
4 N_{0} \lim _{T \uparrow T_{c}} T \int_{\varepsilon}^{\hbar \omega_{D}} \frac{1}{T_{c}-T} \ln \frac{1+e^{-\sqrt{\xi^{2}+u(T, \xi)^{2}} / T}}{1+e^{-\xi / T}} d \xi=-2 N_{0} \int_{\varepsilon}^{\hbar \omega_{D}} \frac{v(\xi)}{\xi} \frac{1}{e^{\xi / T_{c}}+1} d \xi .
$$

We thus see that $\Psi$ is differentiable at $T=T_{c}$ and that

$$
\lim _{T \uparrow T_{c}} \frac{\Psi\left(T_{c}\right)-\Psi(T)}{T_{c}-T}=0 .
$$

A straightforward calculation gives the following results.

LEMMA 4.4. Let $g$ be as in (2.4). Then $g \in C^{1}[0, \infty)$, and

$$
g(\eta)<0 \quad(\eta \geq 0), \quad g^{\prime}(0)=0, \quad \lim _{\eta \rightarrow \infty} g(\eta)=\lim _{\eta \rightarrow \infty} g^{\prime}(\eta)=0 .
$$

Lemma 4.5. Let $\Psi$ be as in (1.6). Then $\Psi \in C^{2}\left[\tau, T_{c}\right]$, and

$$
\frac{\partial^{2} \Psi}{\partial T^{2}}\left(T_{c}\right)=\frac{N_{0}}{8 T_{c}^{2}} \int_{\varepsilon /\left(2 T_{c}\right)}^{\hbar \omega_{D} /\left(2 T_{c}\right)} v\left(2 T_{c} \eta\right)^{2} g(\eta) d \eta \quad(<0) .
$$

Proof. A straightforward calculation gives that $(\partial \Psi / \partial T)$ is differentiable on $\left[\tau, T_{c}\right)$ and that $\left(\partial^{2} \Psi / \partial T^{2}\right)$ is continuous on $\left[\tau, T_{c}\right)$. So it suffices to show that $(\partial \Psi / \partial T)$ is differentiable at $T=T_{c}$ and that $\left(\partial^{2} \Psi / \partial T^{2}\right)$ is continuous at $T=T_{c}$. Note that $(\partial \Psi / \partial T)\left(T_{c}\right)=0$ by Lemma 4.3. Then

$$
\begin{aligned}
& \frac{\frac{\partial \Psi}{\partial T}\left(T_{c}\right)-\frac{\partial \Psi}{\partial T}(T)}{T_{c}-T} \\
& =-N_{0} \int_{\varepsilon}^{\hbar \omega_{D}} \frac{u(T, \xi)^{2} u(T, \xi) \frac{\partial u}{\partial T}(T, \xi)}{\left(T_{c}-T\right)\left(\xi^{2}+u(T, \xi)^{2}\right)}\left\{\frac{1}{2 T \cosh ^{2}\left(\sqrt{\xi^{2}+u(T, \xi)^{2}} / 2 T\right)}\right. \\
& \left.\quad-\frac{\tanh \left(\sqrt{\xi^{2}+u(T, \xi)^{2}} / 2 T\right)}{\sqrt{\xi^{2}+u(T, \xi)^{2}}}\right\} d \xi \\
& \quad+N_{0} \int_{\varepsilon}^{\hbar \omega_{D}} \frac{u(T, \xi)^{2}}{T_{c}-T} \frac{1}{2 T^{2} \cosh ^{2}\left(\sqrt{\xi^{2}+u(T, \xi)^{2}} / 2 T\right)} d \xi
\end{aligned}
$$




$$
\begin{aligned}
& +4 N_{0} \int_{\varepsilon}^{\hbar \omega_{D}} \frac{1}{T_{c}-T} \ln \frac{1+e^{-\sqrt{\xi^{2}+u(T, \xi)^{2}} / T}}{1+e^{-\xi / T}} d \xi \\
& +4 N_{0} \int_{\varepsilon}^{\hbar \omega_{D}} \frac{1}{T_{c}-T}\left\{\frac{\sqrt{\xi^{2}+u(T, \xi)^{2}} / T}{e^{\sqrt{\xi^{2}+u(T, \xi)^{2}} / T}+1}-\frac{\xi / T}{e^{\xi / T}+1}\right\} d \xi .
\end{aligned}
$$

By $(\mathrm{C} 2)$ of Condition $(\mathrm{C})$, for an arbitrary $(0<) \varepsilon_{1}<1$, there is a $\delta>0$ such that $\left|T_{c}-T\right|<\delta$ implies

$$
\left|\frac{u(T, \xi)^{2}}{T_{c}-T} \frac{u(T, \xi) \frac{\partial u}{\partial T}(T, \xi)}{\xi^{2}+u(T, \xi)^{2}}\right|<\frac{\left(v(\xi)+T_{c} \varepsilon_{1}\right)^{2}}{2 \xi^{2}}<\frac{\left(v(\xi)+T_{c}\right)^{2}}{2 \xi^{2}} .
$$

The Lebesgue dominated convergence theorem therefore implies that the first term on the right-hand side of (4.3) becomes

$$
\frac{N_{0}}{2} \int_{\varepsilon}^{\hbar \omega_{D}} \frac{v(\xi)^{2}}{\xi^{2}}\left\{\frac{1}{2 T_{c} \cosh ^{2}\left(\xi / 2 T_{c}\right)}-\frac{\tanh \left(\xi / 2 T_{c}\right)}{\xi}\right\} d \xi
$$

as $T \uparrow T_{c}$. Similarly, the rest of the right-hand side of (4.3) becomes zero as $T \uparrow T_{c}$. We thus find that $(\partial \Psi / \partial T)$ is differentiable at $T=T_{c}$ and that

$$
\frac{\partial^{2} \Psi}{\partial T^{2}}\left(T_{c}\right)=\frac{N_{0}}{2} \int_{\varepsilon}^{\hbar \omega_{D}} \frac{v(\xi)^{2}}{\xi^{2}}\left\{\frac{1}{2 T_{c} \cosh ^{2}\left(\xi / 2 T_{c}\right)}-\frac{\tanh \left(\xi / 2 T_{c}\right)}{\xi}\right\} d \xi
$$

Continuity of $\left(\partial^{2} \Psi / \partial T^{2}\right)$ at $T=T_{c}$ follows immediately.

Our proof of Theorem 2.4 is complete. Moreover, Proposition 2.5 follows immediately from Remark 1.11 and Lemma 4.5.

\section{REFERENCES}

[1] V. Bach, E. H. Lieb and J. P. Solovej. Generalized Hartree-Fock theory and the Hubbard model. J. Stat. Phys. 76 (1994), 3-89.

[2] J. Bardeen, L. N. Cooper and J. R. Schrieffer. Theory of superconductivity. Phys. Rev. 108 (1957), 1175-1204.

[3] P. Billard and G. Fano. An existence proof for the gap equation in the superconductivity theory. Commun. Math. Phys. 10 (1968), 274-279.

[4] N. N. Bogoliubov. A new method in the theory of superconductivity I. Sov. Phys. JETP 34 (1958), 41-46.

[5] R. L. Frank, C. Hainzl, S. Naboko and R. Seiringer. The critical temperature for the BCS equation at weak coupling. J. Geom. Anal. 17 (2007), 559-568.

[6] C. Hainzl, E. Hamza, R. Seiringer and J. P. Solovej. The BCS functional for general pair interactions. Commun. Math. Phys. 281 (2008), 349-367.

[7] C. Hainzl and R. Seiringer. Critical temperature and energy gap for the BCS equation. Phys. Rev. B 77 (2008), 184517.

[8] A. L. Kuzemsky. Bogoliubov's vision: quasiaverages and broken symmetry to quantum protectorate and emergence. Int. J. Mod. Phys. B 24 (2010), 835-935.

[9] A. L. Kuzemsky. Variational principle of Bogoliubov and generalized mean fields in many-particle interacting systems. Int. J. Mod. Phys. B 29 (2015), 1530010.

[10] A. L. Kuzemsky. Statistical Mechanics and the Physics of Many-Particle Model Systems. World Scientific, Singapore, 2017.

[11] M. Niwa. Fundamentals of Superconductivity (in Japanese). Tokyo Denki University Press, Tokyo, 2002.

[12] F. Odeh. An existence theorem for the BCS integral equation. IBM J. Res. Develop. 8 (1964), 187-188.

[13] A. Vansevenant. The gap equation in the superconductivity theory. Physica 17D (1985), 339-344.

[14] S. Watanabe. The solution to the BCS gap equation and the second-order phase transition in superconductivity. J. Math. Anal. Appl. 383 (2011), 353-364. 
[15] S. Watanabe. Addendum to 'The solution to the BCS gap equation and the second-order phase transition in superconductivity'. J. Math. Anal. Appl. 405 (2013), 742-745.

[16] S. Watanabe. An operator-theoretical treatment of the Maskawa-Nakajima equation in the massless abelian gluon model. J. Math. Anal. Appl. 418 (2014), 874-883.

[17] J. M. Ziman. Principles of the Theory of Solids. Cambridge University Press, Cambridge, 1972.

\author{
Shuji Watanabe \\ Division of Mathematical Sciences \\ Graduate School of Engineering \\ Gunma University \\ 4-2 Aramaki-machi \\ Maebashi 371-8510 \\ Japan
}

(E-mail: shuwatanabe@gunma-u.ac.jp) 